\title{
MEDIDAS AUTOMATIZADAS DE PSICRÔMETRO DE TERMOPAR ASPIRADO VERSUS NÃO ASPIRADO
}

\author{
ANTONIO RIBEIRO DA CUNHA E CLOVIS ALBERTO VOLPE
}

\author{
Universidade Estadual Paulista, Faculdade de Ciências Agronômicas, Departamento de Solos e Recursos \\ Ambientais, Botucatu, SP, Brasil \\ arcunha@fca.unesp.br
}

Recebido Novembro de 2012 - Aceito Agosto de 2013

\begin{abstract}
RESUMO
O termopar ou par termoelétrico é utilizado na obtenção da temperatura do ar com ótima precisão, sensibilidade e com grande vantagem sobre o termômetro de mercúrio ou álcool, pois geram sinais elétricos que podem ser armazenados em sistemas automatizados. No entanto, quando se trata da obtenção da umidade relativa a partir de termopar, o processo se torna mais cauteloso devido a outros fatores envolvidos. Neste estudo, avaliaram-se dois conjuntos psicrômetros de termopar, aspirado e não aspirado, em relação a um conjunto Vaisala, no período de 17 a 31/01/2008 (15 dias). A partir de medidas de temperaturas de bulbos seco e úmido foram obtidas medidas da umidade relativa, e com isso, foi possível compará-las com as medidas obtidas pelo conjunto Vaisala. Os dois tipos de psicrômetros apresentaram precisão e exatidão nas medidas de temperatura do ar, e apenas precisão nas medidas de umidade relativa do ar, sendo que o psicrômetro aspirado foi o mais preciso. Apesar do psicrômetro não aspirado ter apresentado menor precisão nas medidas, ele é mais robusto porque independe do micro-ventilador de $12 \mathrm{VDC}$, o que faz dele um sensor com menos riscos de medidas inadequadas. Ambos os psicrômetros, aspirado e não aspirado, não medem com exatidão a umidade relativa do ar no período noturno.
\end{abstract}

Palavras-chave: sistema de aquisição de dados, termopar, temperatura e umidade relativa do ar.

\section{ABSTRACT: AUTOMATIC MEASUREMENTS OF ASPIRATED THERMOCOUPLE PSYCHROMETERS VERSUS NO-ASPIRATED}

The thermocouple is used for obtaining the air temperature with sensitivity and optimum precision, beyond great advantage over the mercury or alcohol thermometer, because it generates electrical signals that can be stored in automated systems. However, when it comes to obtaining the relative humidity from thermocouple, it is needed caution due to other factors involved. In this study two sets of thermocouple psychrometers, aspirated and no-aspirated were evaluated, relative to a Vaisala set, from 17 to 31/01/2008 (15 days). From measurements of dry and wet bulb temperatures, the relative humidity value was obtained, and it was possible to compare them with the Vaisala set measurements. The two types of psychrometers showed precision and accuracy in measurements of air temperature, and only precision in measurements of relative humidity, being that the aspirated psychrometer presented more precision. Despite the no-aspirated psychrometer has lesser precision in measurements, it is more robust because it is independent of a 12 VDC micro-fan, making it, a sensor with lesser risk of inadequate measures. Both aspirated and no-aspirated psychrometers do not measure accurately the relative air humidity during night period.

Keywords: datalogger, thermocouple, air temperature and relative humidity. 


\section{INTRODUÇÃO}

O psicrômetro é um instrumento constituído de dois termômetros, um de bulbo seco e outro de bulbo úmido. O de bulbo seco mede a temperatura real do ar, enquanto que o de bulbo úmido envolto por um cordão de algodão sempre saturado que perde água a uma taxa que depende da concentração de vapor na atmosfera, mede uma temperatura sempre menor ou no máximo igual à temperatura do bulbo seco. Quanto maior a diferença entre os dois termômetros (diferença psicrométrica) maior o poder evaporante do ar, e consequentemente, menor a umidade relativa do ar, sendo um instrumento muito utilizado para a determinação do conteúdo do vapor d'água da atmosfera (Fritschen e Gay, 1979; White e Ross, 1991).

O termopar ou par termoelétrico é utilizado para a obtenção da temperatura do ar com ótima precisão, sensibilidade e com grande vantagem sobre o termômetro de mercúrio ou álcool, pois geram sinais elétricos que podem ser armazenados em sistemas automatizados (datalogger). Além disso, pode ser usado em longas distâncias sem afetar o sinal (Pearcy et al., 1989), sendo o termopar tipo $\mathrm{T}$ (cobre-constantan) o mais adequado para estudos biológicos (Dorcas e Peterson, 2013), por ser simples na forma de utilização, robusto e de baixo custo.

Um dos maiores problemas enfrentados para quem manuseia esse tipo de equipamento, é com relação ao sistema de saturação do termopar de bulbo úmido, que deve ser contínuo. Outro seria com relação à corrente forçada de ar no tipo aspirado para uniformizar o fluxo de ar para as medidas de bulbos seco e úmido, que irá permitir a evaporação da água contida no cordão do termopar de bulbo úmido. Segundo Bindon (1965) recomenda-se a fixação de um micro-ventilador para aspirar o ar de forma constante, e assim obter medidas estabilizadas e corretas das temperaturas de bulbos seco e úmido, pois caso isso não seja feito, medidas erradas de bulbos seco e úmido serão obtidas, e consequentemente, de umidade relativa do ar.

Este trabalho teve como objetivo avaliar e comparar as medidas de temperatura e umidade relativa do ar a partir de dois diferentes conjuntos psicrométricos de termopares de cobre-constantan, um aspirado e outro não aspirado, em relação às medidas de um conjunto Vaisala.

\section{MATERIAL E MÉTODOS}

As medidas foram obtidas numa área experimental próxima $200 \mathrm{~m}$ da Estação Agrometeorológica da Faculdade de Ciências Agrárias e Veterinárias, UNESP, Jaboticabal, SP (latitude de $21^{\circ} 14^{\prime} 05^{\prime}$ " S, longitude de $48^{\circ} 17^{\prime} 09^{\prime \prime}$ W e altitude de $615 \mathrm{~m}$ ).

Foram utilizados dois tipos de psicrômetros, não aspirado (PN) e aspirado (PA), os quais foram construídos com tecnologia e material relativamente simples, utilizando-se termopares do tipo T (cobre-constantan) de bitola 2x24 AWG, em que foram verificadas as medidas em dois estados físicos da água, para gelo fundente $\left(0,2^{\circ} \mathrm{C}\right)$ e água em ebulição $\left(99,6^{\circ} \mathrm{C}\right)$.

O PN foi confeccionado com placas de PVC branco de forma que formasse uma veneziana sobreposta com forma triangular, com intuito de proteger os termopares de bulbo seco e úmido da radiação solar direta e permitisse boa aeração natural - não aspirado. Fixado a essas placas, um tubo de PVC branco de $1,27 \mathrm{~cm}$ de diâmetro e com $20 \mathrm{~cm}$ de comprimento para inserir os termopares e prender o reservatório de água (Cunha et al., 2001; PN, Figura 1).

Já o PA foi confeccionado com tubos de PVC branco de 2" e 4", dois "caps" de 4", "cooler" de computador de 12 VDC (50x50x10 mm - $3500 \mathrm{RPM})$ e reservatório de água. O tubo menor (2") é inserido dentro do maior (4"), e os dois “caps" são usados para fixá-los, sendo feitos 6 furos de $5 \mathrm{~mm}$ para a dissipação de calor. Acoplou-se o "cooler" 12 VDC de forma que aspirasse o ar do tubo menor (2") tipo duto, o qual era ligado por 1 minuto para garantir um fluxo de ar constante e a estabilidade das medidas dos termopares de bulbos seco e úmido no momento da leitura (Marin et al., 2001; PA, Figura 1).

A obtenção da temperatura foi feita conforme a primeira Lei Termoelétrica, ou seja, a força eletromotriz $(\varepsilon)$ de um termopar depende da natureza dos condutores e da diferença de temperatura entre as junções de contato, segundo a Equação 1:

$$
\varepsilon=k\left(T_{a r}-T_{r e f}\right)
$$

em que: $\varepsilon$ é força eletromotriz gerada pelos termopares, em $\mathrm{mV}$, $k$ a constante termoelétrica que depende do tipo de termopar, em $\mu \mathrm{V}^{\circ} \mathrm{C}^{-1}, T_{a r}$ que neste caso para o termopar cobre-constantan

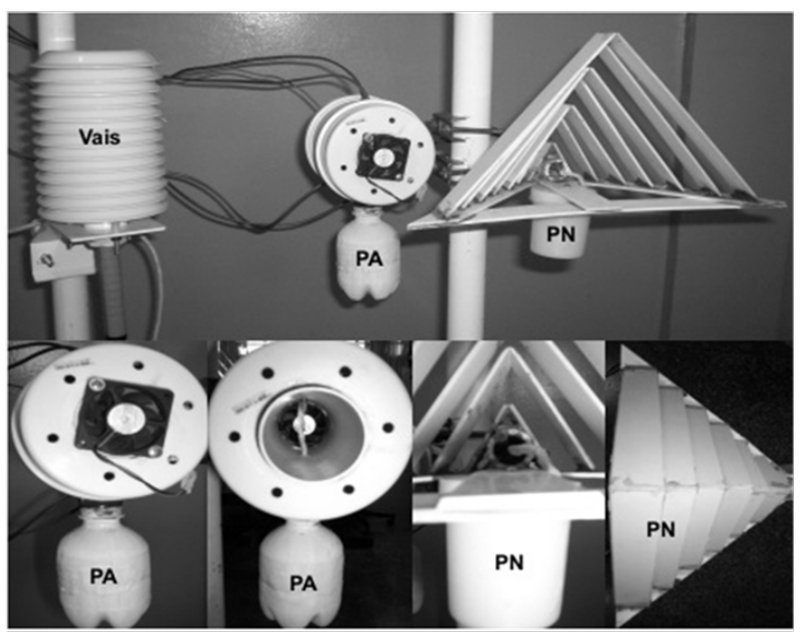

Figura 1 - Fotografia identificando os conjuntos (sensores + abrigo): Vaisala - Vais (termohigrômetro HMP50 + multi-placa RM Young model 41002); psicrômetro aspirado - PA (termopares + abrigo tipo duto) e psicrômetro não aspirado - PN (termopares + abrigo tipo veneziana). Foto: Cunha, (2008). 
é de $41 \mu \mathrm{V}^{\circ} \mathrm{C}^{-1}$, é a temperatura que se deseja medir, em ${ }^{\circ} \mathrm{C}$, e $T_{\text {ref }}$ a temperatura de referência, em ${ }^{\circ} \mathrm{C}$.

Com os termopares de cobre-constantan de bulbos seco e úmido foi possível obter a umidade relativa do ar por meio de relações psicrométricas (Equações 2, 3 e 4):

$$
U R=\frac{e_{a}}{e_{s}} 100
$$

onde:

$$
\begin{aligned}
& e_{a}=e_{s u}-A P\left(t_{s}-t_{u}\right) \\
& e_{s u}\left(t_{u}\right) ; e_{s}\left(t_{s}\right)=0,611 \cdot 10^{\left(\frac{7,5 . t}{237,3+t}\right)}
\end{aligned}
$$

em que: $U R$ é a umidade relativa do ar, em $\%, e_{a}$ a pressão parcial de vapor d'água, em $\mathrm{kPa}, e_{S}$ a pressão de saturação de vapor d'água à temperatura indicada pelo termômetro bulbo $\operatorname{seco}\left(t_{s}\right)$, em $\mathrm{kPa}, e_{s u}$ a pressão de saturação de vapor d'água à temperatura indicada pelo termômetro bulbo úmido $\left(t_{u}\right)$, em $\mathrm{kPa}$, $A$ a constante psicrométrica para o psicrômetro com aspiração forçada $\left(67.10^{-50} \mathrm{C}^{-1}\right)$ e natural $\left(80.10^{-50} \mathrm{C}^{-1}\right), P$ a pressão atmosférica do local $(94,36 \mathrm{kPa}), t_{s}$ a temperatura indicada pelo termômetro de bulbo seco, em ${ }^{\circ} \mathrm{C}$, e $t_{u}$ a temperatura indicada pelo termômetro de bulbo úmido, em ${ }^{\circ} \mathrm{C}$.

Para efeito comparativo em relação aos conjuntos psicrômetros, utilizou-se um conjunto Vaisala (Vais; termohigrômetro HMP50 + multi-placa RM Young model 41002). O Vais é usado como referência para a calibração de instrumentos que medem a UR (Allen et al., 1994) por apresentar alta qualidade metrológica e fazer parte da maioria das estações automáticas (WMO, 2008). Os conjuntos psicrômetros de termopar, PA e PN, e o Vais foram instalados a $2 \mathrm{~m}$ de altura em três locais diferentes (1, 2 e 3$)$.

Foram feitas medidas contínuas no período de 17 a 31/01/2008 (15 dias) de temperaturas de bulbos seco e úmido dos $\mathrm{PN}$ e PA, e da temperatura e umidade relativa do ar pelo Vais, com varredura a cada minuto e armazenamento dos dados médios a cada 10 minutos, a partir de três sistemas de aquisição de dados independentes modelo CR10X (CAMPBELL SCI, 1986-2001).

A comparação dos valores a cada 10 minutos, de temperatura e/ou umidade relativa do ar obtida pelos PA e/ou PN em relação ao Vais, foi feita utilizando-se o teste $t$ ao nível de $5 \%$ de probabilidade para o teste de médias, e também utilizando os seguintes indicadores estatísticos: análise de regressão coeficiente de determinação $\left(\mathrm{R}^{2}\right)$, índice de concordância (d), erro máximo absoluto (ME), erro médio absoluto (MBE) e eficiência (EF), segundo Willmott et al. (1985):

$$
d=1-\left[\frac{\sum_{i=1}^{n}\left(O_{i}-E_{i}\right)^{2}}{\sum_{i=1}^{n}\left(\left|O_{i}-\bar{E}\right|+\left|E_{i}-\bar{E}\right|\right)^{2}}\right]
$$

$$
\begin{aligned}
& M E=\max \left(\left|E_{i}-O_{i}\right|\right)_{i=1}^{n} \\
& M B E=\frac{1}{n} \sum_{i=1}^{n}\left(E_{i}-O_{i}\right) \\
& E F=\frac{\sum_{i=1}^{n}\left(O_{i}-\bar{O}\right)^{2}-\sum_{i=1}^{n}\left(O_{i}-E_{i}\right)^{2}}{\sum_{i=1}^{n}\left(O_{i}-\bar{O}\right)^{2}}
\end{aligned}
$$

em que $E_{i}$ é o valor da temperatura e/ou umidade relativa do ar obtido pelo psicrômetro de termopar (PA ou PN), $O_{i}$ o valor da temperatura e/ou umidade relativa do ar obtido pelo Vais, $\bar{E}$ a média da temperatura e/ou umidade relativa do ar obtida pelo psicrômetro de termopar (PA ou PN), $\bar{O}$ a média da temperatura e/ou umidade relativa do ar obtida pelo Vais, e $n$ o número de observações. Se $E_{i}$ é igual a $O_{i}$ tem-se que:

$$
M E=M B E \text { e } R^{2}=d=E=1 .
$$

\section{RESULTADOS E DISCUSSÃO}

Selecionou-se três dias seguidos, DJ44 (parcialmente límpido), DJ 45 (parcialmente nublado) e DJ46 (nublado) para cada um dos três locais analisados (1, 2 e 3 ), em que DJ é o dia Juliano. Foram analisados os dados médios a cada $10 \mathrm{~min}$., para avaliar a variação da temperatura (T) (Figura 2) e umidade relativa (UR) (Figura 3) nos dois conjuntos de psicrômetros, aspirado (PA) e/ou não aspirado (PN) em relação ao conjunto Vaisala (Vais).

Com relação à T (Figura 2), observou-se que as diferenças em relação ao Vais foram maiores durante o período diurno, entre as $12 \mathrm{~h} 00 \mathrm{~min}$ e $17 \mathrm{~h} 00 \mathrm{~min}$. No DJ44 as maiores diferenças de $\mathrm{T}$ foram do PN no local $1\left(4,0^{\circ} \mathrm{C}\right)$, local $2\left(4,5^{\circ} \mathrm{C}\right)$ e local 3 $\left(3,1^{\circ} \mathrm{C}\right)$. No DJ45 as maiores diferenças também foram do PN, com $6,2{ }^{\circ} \mathrm{C}$ (local 1), $6,9^{\circ} \mathrm{C}$ (local 2) e $4,6{ }^{\circ} \mathrm{C}$ (local 3). Já no DJ46 as maiores diferenças foram do PA no local $1\left(3,4^{\circ} \mathrm{C}\right)$, do PN no local $2\left(6,0^{\circ} \mathrm{C}\right)$ e no local $3\left(3,7^{\circ} \mathrm{C}\right)$. Ficou claramente demonstrado que o PA foi mais semelhante ao Vais nas medidas de T, considerando a maioria dos dias e locais analisados.

Com relação à UR (Figura 3), observou-se que as diferenças dos PA e/ou PN em relação ao Vais foram maiores durante o período noturno, entre as $18 \mathrm{~h} 00 \mathrm{~min}$ e $07 \mathrm{~h} 00 \mathrm{~min}$. Essas maiores diferenças ocorridas no período noturno deve-se ao fato de que a água contida no reservatório dos conjuntos psicrômetros (PA e PN) não acompanha o resfriamento noturno da atmosfera. A água contida no reservatório armazena calor ao longo do dia, e quando chega a noite, a perda desse calor é mais lenta do que a que ocorre na atmosfera, devido ao calor específico da água ser alto, o que faz com que a água não apresente variações bruscas em sua temperatura. Neste caso, a água do reservatório está a uma temperatura acima da qual deveria estar realmente, o que 

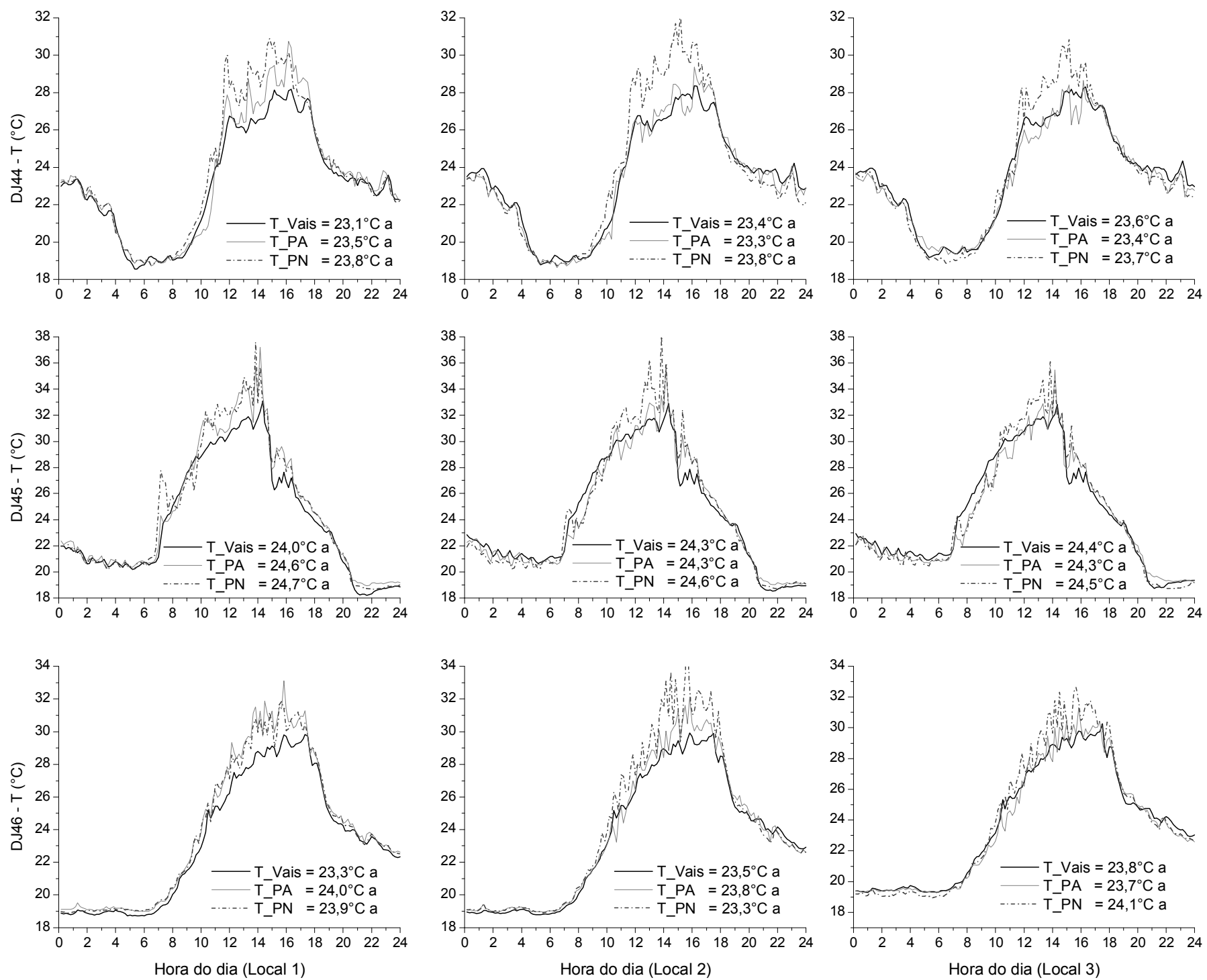

Figura 2 - Variação da temperatura (T) nos locais 1, 2 e 3 para os três conjuntos: Vaisala (Vais), psicrômetro aspirado (PA) e psicrômetro não aspirado (PN), para os dias julianos DJ44, DJ45 e DJ46 de 2008. Jaboticabal, SP.

vai diminuir o processo de troca de calor entre o termômetro de bulbo úmido e a atmosfera, ocasionando uma diferença psicrométrica menor, e consequentemente, valores maiores de UR em relação ao conjunto Vais no período noturno.

No DJ44 as maiores diferenças na UR foram do PA com 19,3\% (local 1) e 23,1\% (local 3), e do PN com 15,2\% (local 2). No DJ45 as maiores diferenças também foram do PN, com $18,2 \%$ (local 1) e $15,7 \%$ (local 2), e do PA com $24,8 \%$ (local 3). Já no DJ46 as maiores diferenças foram do PN, com 16,6\% (local 1) e 13,8\% (local 2), e do PA com 26,6\% (local 3). Isso demonstrou que o PN foi mais semelhante ao Vais nas medidas de UR, considerando a maioria dos dias e locais analisados.

Em todos os casos, nos dias e locais analisados, os conjuntos PA e PN diferiram estatisticamente do Vais, superestimando os valores de UR em relação ao Vais. Apenas no local 3 houve as maiores diferenças de UR durante o período diurno, para os dois conjuntos, PA e PN, sendo que para o PA a diferença foi maior, com ambos diferindo estatisticamente do Vais. Considerando a medida (T, UR) em cada local e obtida pelos três diferentes sensores, observou-se para a medida de $\mathrm{T}$, que no local 1 os sensores PA e PN foram semelhantes entre si e diferentes em relação ao Vais. No local 2 o PA assemelhou-se ao Vais, sendo que Vais e PA foram diferentes em relação ao PN. Já no local 3, tanto o PA como o PN assemelharam-se ao Vais, mas o PA foi diferente em relação ao PN. Para a UR, nos locais 1 e 3 , todos os sensores foram diferentes entre si, enquanto que no local 2, os sensores PA e PN foram semelhantes entre si e diferentes em relação ao Vais (Tabela 1). 

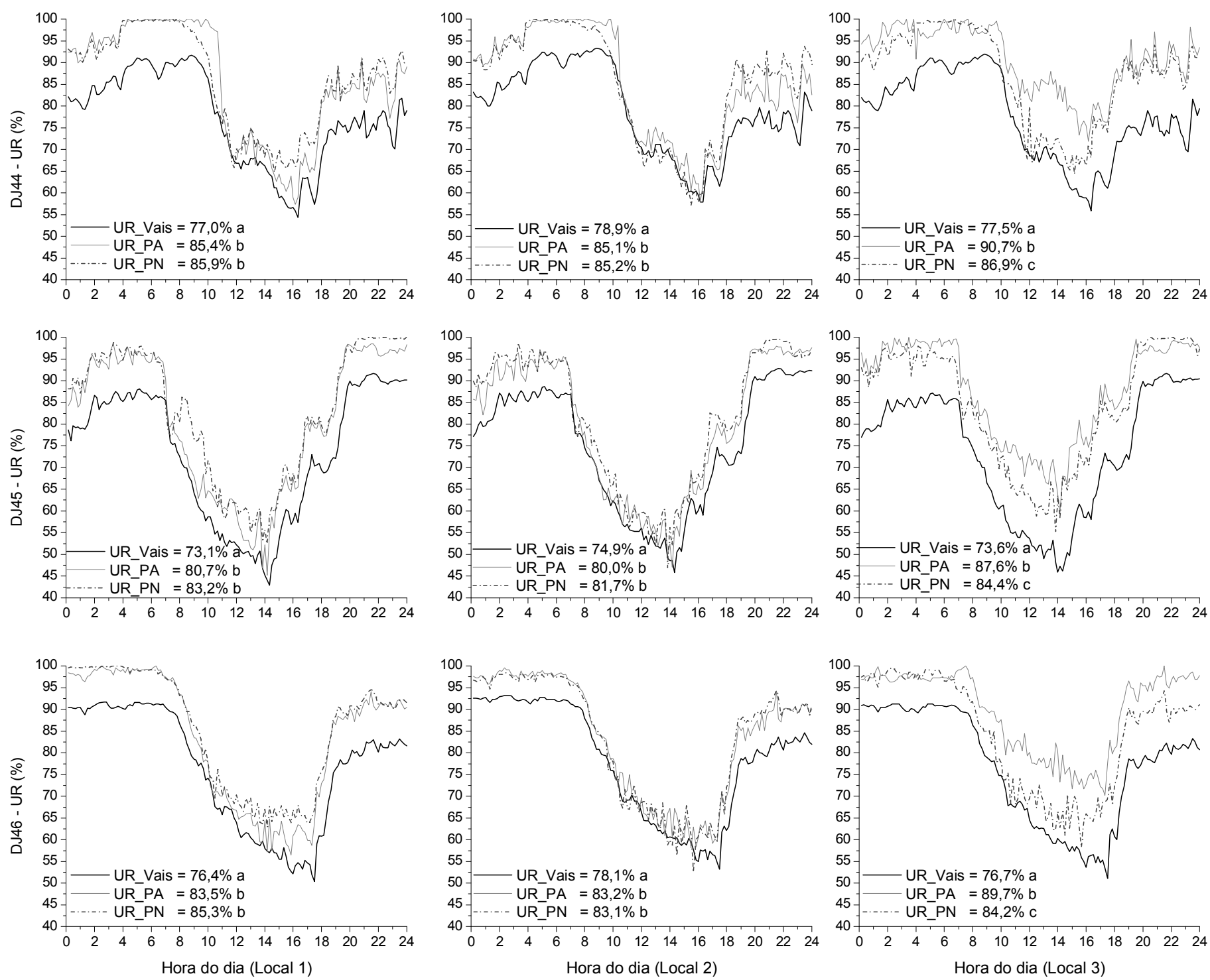

Figura 3 -Variação da umidade relativa (UR) nos locais 1, 2 e 3 para os três conjuntos: Vaisala (Vais), psicrômetro aspirado (PA) e psicrômetro com ventilação natural (PN), para os dias julianos DJ44, DJ45 e DJ46 de 2008. Jaboticabal, SP.

Considerando a medida (T, UR) entre os locais e obtida pelo mesmo sensor, observou-se que não houve semelhança na medida de T para o sensor Vais entre os locais 1, 2 e 3, enquanto que os sensores PA e PN apresentaram semelhanças nas medidas nos três locais. Para a medida de UR, os sensores Vais e PN apresentaram semelhança apenas nos locais 1 e 3, enquanto que o PA apresentou semelhança nos locais 1 e 2 (Tabela 1).

Praticamente em todos os locais os valores de T e UR medidos a partir dos sensores PA e PN foram maiores em relação ao sensor Vais, com exceção do local 3 para medida de $\mathrm{T}$ (Tabelas 2 e 3; Figuras 4, 5 e 6 ).

A qualidade de medida da T e da UR depende da precisão e exatidão. A precisão representa a dispersão das medidas em relação a um valor médio associado ao erro padrão $\left(\mathrm{R}^{2} \mathrm{e} d\right)$, enquanto que a exatidão assegura que a medida coincida com o valor real da grandeza considerada (MBE e ME).

A exatidão está associada ao MBE em relação a um sensor referência, que neste caso foi o Vais, e, portanto, o sensor PA foi mais de acordo com o Vais no local 3 (MBE $=0,10$ e $\mathrm{ME}=3,43)$ para medidas de $\mathrm{T}$, e no local $2(\mathrm{MBE}=5,40 \mathrm{e}$ $\mathrm{ME}=29,65$ ) para medidas de UR (Tabela 2). O mesmo aconteceu com o sensor PN, que foi mais de acordo com o Vais no local $3(\mathrm{MBE}=0,17$ e $\mathrm{ME}=4,63)$ para medidas de $\mathrm{T}$, e no local 2 $(\mathrm{MBE}=5,25$ e $\mathrm{ME}=32,07)$ para medidas de UR (Tabela 3).

Pelas Tabelas 2 e 3, e Figuras 3 e 5, o local 2 foi o que melhor representou o comportamento da medida da UR para os dois conjuntos de psicrômetros analisados (PA e PN), pois apresentou MBE em torno de 5\% para faixa de medida da UR, 
Tabela 1 - Valores médios de temperatura $\left(\mathrm{T},{ }^{\circ} \mathrm{C}\right)$ e umidade relativa (UR, \%) obtidos pelos sensores Vaisala (Vais), psicrômetro de termopar aspirado (PA) e não aspirado (PN). Jaboticabal, SP.

\begin{tabular}{|c|c|c|c|c|c|c|c|}
\hline \multirow{2}{*}{$\begin{array}{l}\text { Locais/ } \\
\text { Sensor }\end{array}$} & \multirow{2}{*}{$\begin{array}{c}\text { Tamanho } \\
\mathrm{N}\end{array}$} & \multicolumn{3}{|c|}{$\mathrm{T}\left({ }^{\circ} \mathrm{C}\right)$} & \multicolumn{3}{|c|}{ UR (\%) } \\
\hline & & Média & IC & $\mathrm{s}$ & Média & IC & $\mathrm{s}$ \\
\hline \multicolumn{8}{|l|}{ Local 1} \\
\hline Vais & 1984 & $23,4 \mathrm{aA}$ & $\pm 0,16$ & 3,60 & 77,8 aA & $\pm 0,61$ & 13,95 \\
\hline$P A$ & 1984 & 23,9 bD & $\pm 0,18$ & 4,04 & 84,9 bC & $\pm 0,68$ & 15,35 \\
\hline$P N$ & 1984 & $24,1 \mathrm{bE}$ & $\pm 0,18$ & 4,17 & $86,1 \mathrm{cE}$ & $\pm 0,61$ & 13,80 \\
\hline \multicolumn{8}{|l|}{ Local 2} \\
\hline Vais & 1984 & $23,6 \mathrm{aB}$ & $\pm 0,16$ & 3,56 & $79,4 \mathrm{aB}$ & $\pm 0,59$ & 13,37 \\
\hline$P A$ & 1984 & $23,7 \mathrm{aD}$ & $\pm 0,17$ & 3,78 & $84,8 \mathrm{bC}$ & $\pm 0,65$ & 14,72 \\
\hline$P N$ & 1984 & $24,1 \mathrm{bE}$ & $\pm 0,19$ & 4,38 & $84,7 \mathrm{bF}$ & $\pm 0,65$ & 14,82 \\
\hline \multicolumn{8}{|l|}{ Local 3} \\
\hline Vais & 1984 & $23,9 \mathrm{abC}$ & $\pm 0,15$ & 3,44 & $78,0 \mathrm{aA}$ & $\pm 0,59$ & 13,34 \\
\hline$P A$ & 1984 & $23,7 \mathrm{aD}$ & $\pm 0,16$ & 3,62 & $88,7 \mathrm{bD}$ & $\pm 0,50$ & 11,33 \\
\hline$P N$ & 1984 & $24,0 \mathrm{bE}$ & $\pm 0,18$ & 4,08 & $86,5 \mathrm{cE}$ & $\pm 0,57$ & 12,94 \\
\hline
\end{tabular}

*Valores médios seguidos da mesma letra minúscula (em cada local para os diferentes sensores) e da mesma letra maiúscula (entre os locais para o mesmo sensor) na coluna não diferem entre si ao nível de $5 \%$ pelo teste $\mathrm{t}$. $\mathrm{IC}=$ intervalo de confiança; $\mathrm{s}=$ desvio padrão.

valor dentro da faixa esperada segundo Bell (1996), pois a cada $\pm 1{ }^{\circ} \mathrm{C}$ de mudança na $\mathrm{T}$, a UR pode variar em até $\pm 6,3 \%$. Então, nos locais 1 e 3 , provavelmente as medidas do termopar de bulbo úmido não foram corretas, pois a evaporação da água da mecha de algodão que envolve o bulbo úmido do psicrômetro não conseguiu acompanhar com a mesma rapidez a demanda atmosférica devido à deficiência na capilaridade. Os erros de superestimativa encontrados na UR são devido ao termômetro de bulbo úmido, seja por deficiência na sua aspiração e/ou capilaridade da sua mecha de algodão, prejudicando a troca térmica entre ele e a atmosfera, fazendo reduzir a depressão psicrométrica, e consequentemente, obtendo-se valores de UR maiores que os valores reais.

No local 1 o PA apresentou um MBE menor que o PN para a medida de UR, o que é devido ao mecanismo acoplado (micro-ventilador) no PA, que aspira mantendo um fluxo de ar constante no momento da medida do bulbo úmido, permitindo uma troca de calor mais eficiente que fluxo de ar natural do PN (Blair e Fite, 1964). Já no local 3, quem apresentou um MBE menor foi o $\mathrm{PN}$, devido provavelmente à deficiência na aspiração do PA e/ou na capilaridade, a qual pode não ter sido suficiente para a estabilização da medida do bulbo úmido, e com isso, o PA acaba se comportando pior que o PN. Isto acontece porque o psicrômetro não aspirado depende do fluxo de ar natural de uma amostra de ar, o qual varia ao longo do tempo, podendo ou não favorecer a evaporação (Blair e Fite, 1964), o que permite muitas vezes medida mais real do processo, pois não depende de particularidades do sistema para medir a amostra de ar. O PN está sempre preparado para as medidas sem necessitar da espera para a estabilidade de leitura que ocorre no PA, mas, no entanto, quando a capilaridade está deficiente na mecha de algodão, em qualquer um dos psicrômetros esse processo é comprometido. Portanto, a exatidão da medida de temperatura de bulbo úmido depende da qualidade da água, aspiração da

Tabela 2 - Indicadores estatísticos entre a temperatura $\left(\mathrm{T},{ }^{\circ} \mathrm{C}\right)$ e umidade relativa (UR, \%) obtida a partir da relação entre o psicrômetro aspirado (PA) e o Vaisala (Vais): coeficiente de determinação $\left(\mathrm{R}^{2}\right)$, índice de concordância (d), erro máximo (ME), erro médio absoluto (MBE) e eficiência (EF). Jaboticabal, SP.

\begin{tabular}{|c|c|c|c|c|c|c|c|}
\hline PA $\times$ Vais & $\mathrm{N}$ & $\mathrm{R}^{2}$ & $\mathrm{~d}$ & $\mathrm{ME}$ & MBE & $\mathrm{EF}$ & $\mathrm{T}_{\mathrm{PA}}=\mathrm{b}\left(\mathrm{T}_{\text {Vais }}\right)$ \\
\hline$T\left({ }^{\circ} \mathrm{C}\right)$ & & & & $\left({ }^{\circ} \mathrm{C}\right)$ & $\left({ }^{\circ} \mathrm{C}\right)$ & & $\left({ }^{\circ} \mathrm{C}\right)$ \\
\hline Local 1 & 1984 & 0,9753 & 0,9992 & 4,93 & 0,53 & 0,9988 & $\mathrm{~T}_{\mathrm{PA}}=1,0246\left(\mathrm{~T}_{\text {Vais }}\right)$ \\
\hline Local 2 & 1984 & 0,9755 & 0,9996 & 3,72 & 0,10 & 0,9994 & $\mathrm{~T}_{\mathrm{PA}}=1,0052\left(\mathrm{~T}_{\text {Vais }}\right)$ \\
\hline Local 3 & 1984 & 0,9716 & 0,9995 & 3,43 & 0,10 & 0,9994 & $\mathrm{~T}_{\mathrm{PA}}=0,9965\left(\mathrm{~T}_{\text {Vais }}\right)$ \\
\hline$U R(\%)$ & & & & $(\%)$ & $(\%)$ & & $(\%)$ \\
\hline Local 1 & 1984 & 0,9510 & 0,9922 & 33,63 & 7,14 & 0,9886 & $\mathrm{UR}_{\mathrm{PA}}=1,0912\left(\mathrm{UR}_{\text {Vais }}\right)$ \\
\hline Local 2 & 1984 & 0,9620 & 0,9955 & 29,65 & 5,40 & 0,9936 & $\mathrm{UR}_{\mathrm{PA}}=1,0683\left(\mathrm{UR}_{\text {Vais }}\right)$ \\
\hline Local 3 & 1984 & 0,8839 & 0,9766 & 26,62 & 10,66 & 0,9657 & $\mathrm{UR}_{\mathrm{PA}}=1,1270\left(\mathrm{UR}_{V_{\text {ais }}}\right)$ \\
\hline
\end{tabular}


Tabela 3 - Indicadores estatísticos entre a temperatura $\left(\mathrm{T},{ }^{\circ} \mathrm{C}\right)$ e umidade relativa do ar (UR, \%) obtida a partir da relação entre o psicrômetro não aspirado (PN) e o Vaisala (Vais): coeficiente de determinação $\left(\mathrm{R}^{2}\right)$, índice de concordância (d), erro máximo (ME), erro médio absoluto (MBE) e eficiência (EF). Jaboticabal, SP.

\begin{tabular}{|c|c|c|c|c|c|c|c|}
\hline PN $x$ Vais & $\mathrm{N}$ & $\mathrm{R}^{2}$ & $\mathrm{~d}$ & ME & MBE & $\mathrm{EF}$ & $\mathrm{T}_{\mathrm{PA}}=\mathrm{b}\left(\mathrm{T}_{\mathrm{Vais}}\right)$ \\
\hline$T\left({ }^{\circ} \mathrm{C}\right)$ & & & & $\left({ }^{\circ} \mathrm{C}\right)$ & $\left({ }^{\circ} \mathrm{C}\right)$ & & $\left({ }^{\circ} \mathrm{C}\right)$ \\
\hline Local 1 & 1984 & 0,9648 & 0,9986 & 6,61 & 0,75 & 0,9980 & $\mathrm{~T}_{\mathrm{PN}}=1,0344\left(\mathrm{~T}_{\text {Vais }}\right)$ \\
\hline Local 2 & 1984 & 0,9508 & 0,9986 & 6,86 & 0,55 & 0,9977 & $\mathrm{~T}_{\mathrm{PN}}=1,0274\left(\mathrm{~T}_{\text {Vais }}\right)$ \\
\hline Local 3 & 1984 & 0,9696 & 0,9991 & 4,63 & 0,17 & 0,9987 & $\mathrm{~T}_{\mathrm{PN}}=1,0110\left(\mathrm{~T}_{\text {Vais }}\right)$ \\
\hline$U R(\%)$ & & & & $(\%)$ & $(\%)$ & & $(\%)$ \\
\hline Local 1 & 1984 & 0,9381 & 0,9881 & 35,03 & 8,31 & 0,9832 & $\mathrm{UR}_{\mathrm{PN}}=1,1022\left(\mathrm{UR}_{\text {Vais }}\right)$ \\
\hline Local 2 & 1984 & 0,9204 & 0,9942 & 32,07 & 5,25 & 0,9919 & $\mathrm{UR}_{\mathrm{PN}}=1,0660\left(\mathrm{UR}_{\text {Vais }}\right)$ \\
\hline Local 3 & 1984 & 0,9152 & 0,9847 & 21,85 & 9,25 & 0,9795 & $\mathrm{UR}_{\mathrm{PN}}=1,1035\left(\mathrm{UR}_{\text {Vais }}\right)$ \\
\hline
\end{tabular}
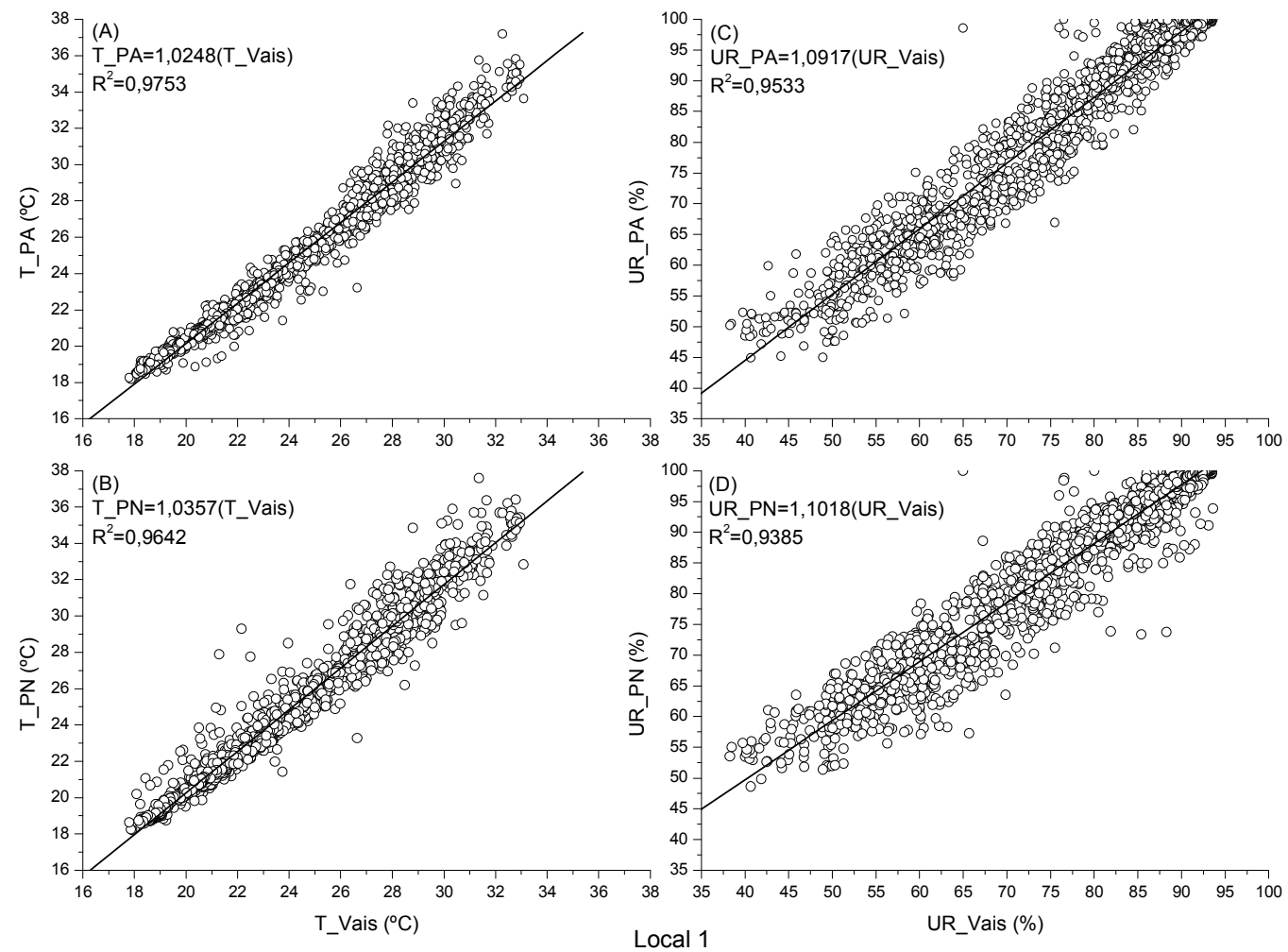

Figura 4 - Correlação entre as T do PA versus Vais (A), T do PN versus Vais (B), UR do PA versus Vais (C), e UR do PN versus Vais (D), para o local 1. Jaboticabal, SP.

corrente de ar, tipo e estado da mecha de algodão e efeitos da radiação solar (ASHRAE STANDARD 41.1, 1994).

De forma geral, o psicrômetro apresenta boa estabilidade nas medidas, mas exige habilidade na manutenção, pois está propenso a erros na medida da temperatura de bulbo úmido, quando a amostra de ar não é umedecida o suficiente pela evaporação da mecha de algodão (Bell, 1996).

Assim, o PA foi o melhor conjunto psicrômetro nas medidas de T e UR, levando-se em conta todos os indicadores estatísticos, o R ${ }^{2}$, d, ME, MBE e EF (Tabelas 2 e 3). Isto está de acordo com Cunha e Martins (2004), pois além da alta concordância ( $\mathrm{R}^{2}$ e d $\left.>0,90\right)$ que está associada com a precisão, numa análise comparativa entre sensores, é importante também que haja um MBE e ME próximos de zero (associados à exatidão) para garantir que ambos os sensores sejam semelhantes entre si nas medidas obtidas.

Neste trabalho, a faixa de medidas da UR variou entre 45 e 100\% (Figuras 4, 5 e 6). É importante destacar que quando os valores de UR são menores que $50 \%$, a demanda atmosférica aumenta muito, e a evaporação da água da mecha de algodão não consegue acompanhar com a mesma rapidez essa demanda devido à deficiência na capilaridade, aumentando o erro na 

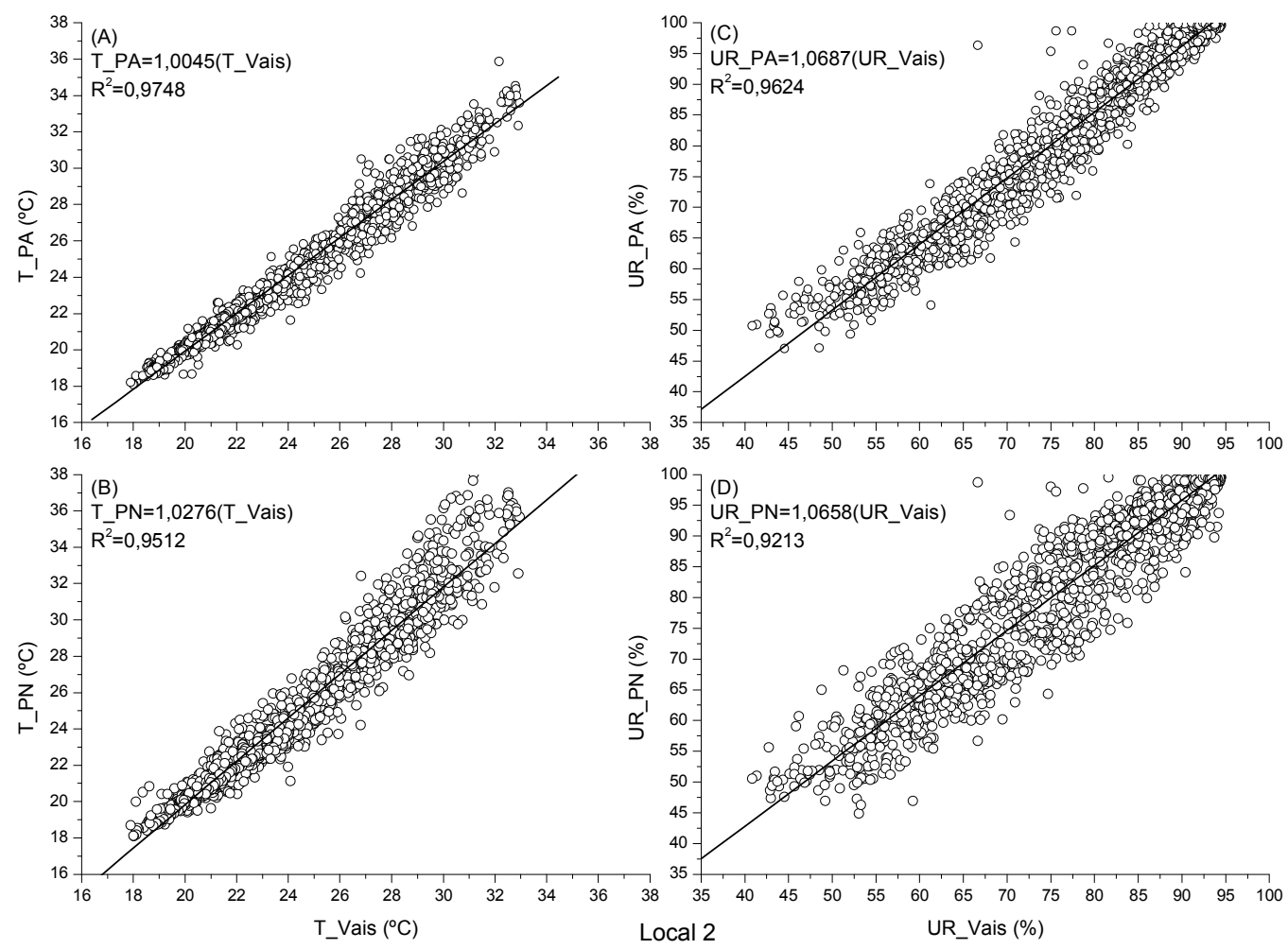

Figura 5 - Correlação entre as T do PA versus Vais (A), T do PN versus Vais (B), UR do PA versus Vais (C), e UR do PN versus Vais (D), para o local 2. Jaboticabal, SP.

medida da UR (Cunha, 2013). Portanto, é importante estar atento às condições da mecha de algodão do bulbo úmido, pois se não houver capilaridade na mecha para mantê-la sempre molhada, medidas erradas de bulbo úmido serão obtidas, e consequentemente de UR. Esse fenômeno físico é afetado pela pureza da água do reservatório, corrente de ar, especificação e estado da mecha, trocas radiantes, efeitos da pressão, e outros (ASHRAE STANDARD 41.1, 1994). Por isso, a mecha deve ser de algodão e estar bem justa ao termopar para evitar erros de medida, além de impurezas que acabam sendo depositadas na mecha ao longo do tempo, necessitando de limpeza ou troca (ASHRAE STANDARD 41.6, 1974). Ainda, os bulbos seco e úmido devem estar protegidos da chuva e da radiação solar por material isolante termicamente, e haver tempo necessário para que o bulbo úmido atinja uma leitura estável em função da taxa de ventilação (ISO, 2007).

A velocidade mínima do ar que resulta na verdadeira temperatura do bulbo úmido é função da sua dimensão e forma (Incropera e DeWitt, 2003), sendo que os termopares requerem baixas velocidades do ar porque apresentam diâmetro pequeno (ISO, 2007). Neste estudo, a velocidade máxima do sistema de aspiração pelo micro-ventilador era em torno de $9 \mathrm{~m} \mathrm{~s}^{-1}$ (3500 RPM), bem acima do recomendado pelo ASHRAE STANDARD 41.6 (1994), que diz que a velocidade deve ser de no mínimo de $1,8 \mathrm{~m} \mathrm{~s}^{-1}$ para as medidas corretas de termopar de bulbo úmido. Ainda, segundo REYES CAC (2005), no psicrômetro de termopar de bulbo úmido do tipo $\mathrm{T}$, para cada incremento de $1 \mathrm{~m} \mathrm{~s}^{-1}$ no valor da velocidade do ar, a constante de tempo diminui em 2,85 seg., sendo assim, o tempo de resposta para uma velocidade do ar de $3 \mathrm{~m} \mathrm{~s}^{-1}$ e uma umidade de $50 \%$ é de 15,53 seg., demonstrando que o psicrômetro de termopar estabiliza rapidamente, ou seja, provê uma rápida resposta de medida. Portanto, o tempo de aspiração utilizado de 1 minuto no PA foi acima do exigido.

No local 1, observa-se que houve uma tendência para superestimativa nos valores de T de $2,48 \%$ para o PA em relação ao Vais (Figura 4A) e de 3,57\% para o PN em relação ao Vais (Figura 4B). Também houve superestimativa nos valores de UR de $9,17 \%$ para o PA em relação ao Vais (Figura 4C) e de 10,18\% para o PN em relação ao Vais (Figura 4D).

No local 2, houve uma tendência para superestimativa nos valores de T de $0,45 \%$ para o PA em relação ao Vais (Figura $5 \mathrm{~A}$ ) e de $2,76 \%$ para o PN em relação ao Vais (Figura 5B). Superestimativa também nos valores de UR de 6,87\% para o PA em relação ao Vais (Figura 5C) e de 6,58\% para o PN em relação ao Vais (Figura 5D).

Para o local 3, houve uma tendência para subestimativa nos valores de T de 0,33\% para o PA em relação ao Vais (Figura $6 \mathrm{~A}$ ) e de $1,14 \%$ para o PN em relação ao Vais (Figura $6 \mathrm{~B}$ ). Houve superestimativa nos valores de UR de $12,68 \%$ para o 

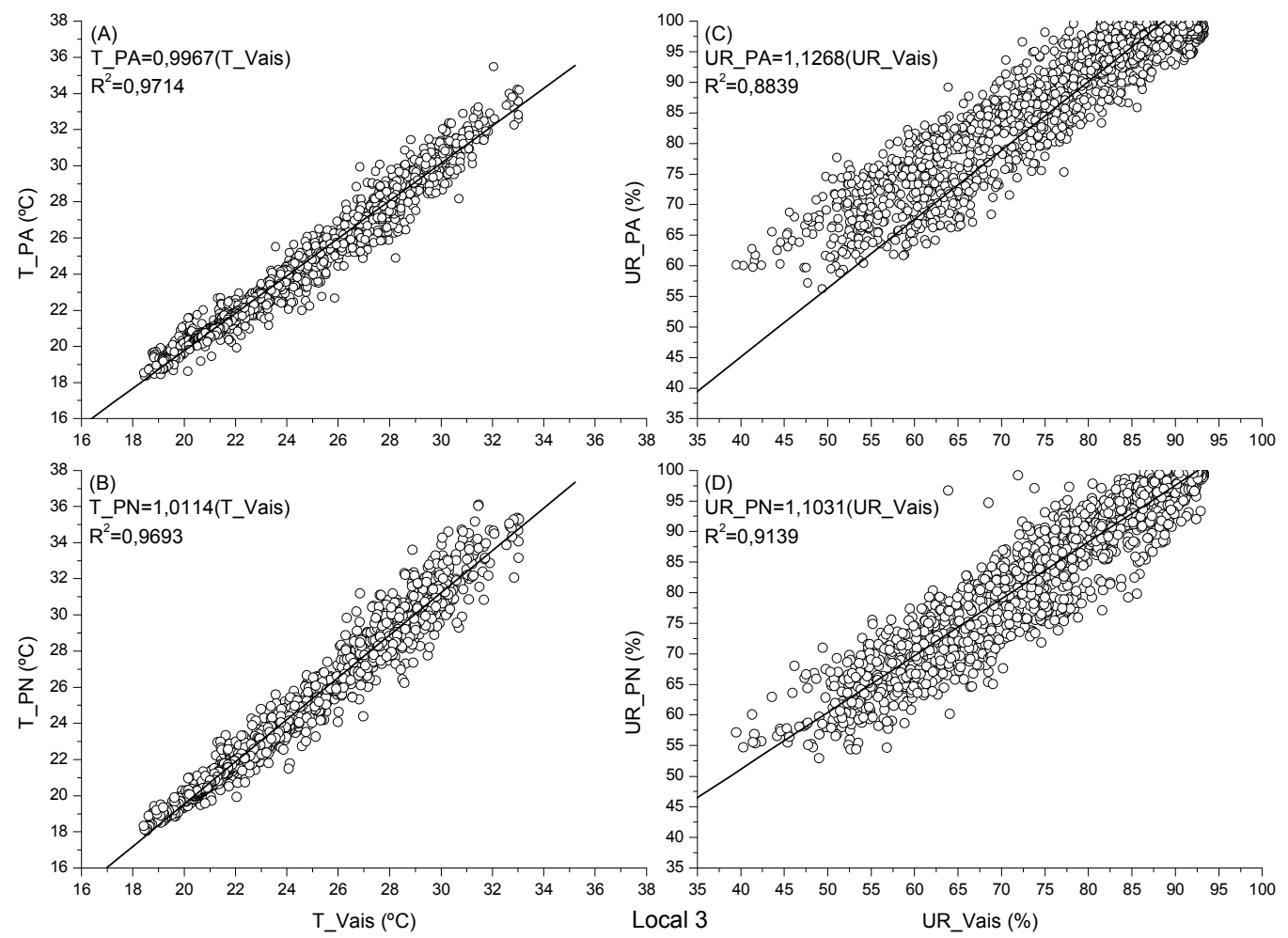

Figura 6 - Correlação entre as T do PA versus Vais (A), T do PN versus Vais (B), UR do PA versus Vais (C), e UR do PN versus Vais (D), para o local 3. Jaboticabal, SP.

PA em relação ao Vais (Figura 6C) e de 10,31\% para o PN em relação ao Vais (Figura 6D).

Autores como Cunha et al. (2001), Marin et al. (2001), Silva et al. (2011) e Cunha (2013) analisando medidas obtidas de psicrômetro de termopar, obtiveram superestimativa na UR. Cunha et al. (2001) analisando dados de 195 dias de um psicrômetro não aspirado, encontraram uma superestimativa de $1,0 \%$ para UR em relação a um sensor Vaisala modelo HMP45C numa faixa de 25 a $100 \%$. Enquanto que Marin et al. (2001), analisando dados de apenas 4 dias de um psicrômetro aspirado, encontraram uma superestimativa de 6,5\% na UR em relação a um sensor Vaisala modelo HMP45C numa faixa de 30 a 90\%. Já Silva et al. (2011), analisando dados de apenas 3 dias encontraram uma superestimativa de $2,7 \%$ na UR obtida por psicrômetro aspirado em relação a um sensor Vaisala modelo HMP45D numa faixa de 55 a 100\%. Enquanto Cunha (2013), analisando dados de 28 dias de dois psicrômetros aspirados englobando dias com temperatura do ar alta e umidade relativa baixa, e dias com umidade relativa do ar alta e temperatura do ar baixa, encontraram uma superestimativa que variaram entre 8-9\% para UR em relação a um sensor Vaisala modelo HMP50 numa faixa de 20 a 100\%, principalmente nos valores abaixo de 50\%. Ainda, segundo Cunha (2013), quando em valores mais baixos da UR (menor que 50\%), a demanda atmosférica aumenta muito, e a evaporação da água da mecha de algodão, que envolve o bulbo úmido do psicrômetro, não consegue acompanhar com a mesma rapidez essa demanda devido à deficiência na capilaridade. Esse fenômeno físico pode ser afetado pela deficiência na ventilação (natural ou forçada) e por eventuais contaminantes na mecha de algodão.

Como os sensores Vais instalados nos três locais estavam adequadamente calibrados para as medidas de T e UR, então, a T obtida pelos dois conjuntos psicrômetros (PA e PN) foi precisa e exata, mas no caso da UR apenas precisa. Assim, apesar do conjunto psicrômetro de termopar ser uma opção mais barata, apresenta uma relativa dificuldade de obter a UR na prática com exatidão (Dias, 2001), isso porque a constituição do psicrômetro envolve componentes que afetam essa medida, tais como: o tipo e qualidade da mecha que envolve o termopar de bulbo úmido para garantir uma capilaridade eficiente, o reservatório de água (tamanho, forma e cor), e o formato do equipamento visando uma ventilação eficiente do sistema.

\section{CONCLUSÕES}

Os dois tipos de psicrômetros de termopar, aspirado e não aspirado, apresentaram precisão e exatidão nas medidas de temperatura do ar, e apenas precisão nas medidas de umidade relativa do ar, sendo que o psicrômetro aspirado foi o mais preciso. 
Apesar do psicrômetro não aspirado ter apresentado menor precisão nas medidas, ele é mais robusto, pois independe do micro-ventilador de 12 VDC, o que faz dele um sensor com menos riscos de medidas inadequadas.

Os dois psicrômetros, aspirado e não aspirado, não medem com exatidão a umidade relativa do ar no período noturno.

\section{AGRADECIMENTOS}

À Fundação de Amparo à Pesquisa do Estado de São Paulo (FAPESP) pela bolsa concedida para a realização do referido trabalho (processo $n^{\circ}$ 05/59535-4).

\section{REFERÊNCIAS BIBLIOGRÁFICAS}

ALLEN, S.J; BRENNER, A.J.; GRACE, J. A lowcost psychrometer form field measurements of atmospheric humidity. Plant, Cell and Environment, v.17, p.219-225, 1994.

ASHRAE STANDARD 41.1 - 1974. Standard measurement guide: section on temperature measurements, ASHRAE, Atlanta. Disponível em: http://www.ashrae.org/publications/. Acesso em: 01 out. 2011.

ASHRAE STANDARD 41.6 - 1994. Methods for measurement of moist air properties, ASHRAE, Atlanta. Disponível em: http://www.ashrae.org/publications/. Acesso em: 01 out. 2011.

BELL, S.A. A guide to the measurement of humidity (NPL Guide 103). Teddington: National Physical Laboratory. 1996.

BINDON, H.H. A critical review of tables and charts use in psychrometry. In: WEXLER, A. Humidity and moisture. New York: Reinhold, 1965, v.1, p.3-15.

BLAIR, T.A., FITE, R.C. Meteorologia. Rio de Janeiro: Ed. Artes Gráficas Gomes de Souza, 1964.

CAMPBELL SCI. CR10X Micrologger: operator's manual. Revision: 9/01. Logan, Utah: Copyright(C), 1986-2001.

CUNHA, A.R Comunicação pessoal. 2008.

CUNHA, A.R. Programação, conexão e avaliação de psicrômetro aspirado de termopar de baixo custo em micrologger para estudos agrometeorológicos. Revista Brasileira de Meteorologia, v.28, p.221-232, 2013.

CUNHA, A.R.; ESCOBEDO, J.F.; GALVANI, E. Avaliação de um psicrômetro de termopar de baixo custo. Revista Brasileira de Agrometeorologia, v.9, p.17-22, 2001.

CUNHA, A.R.; MARTINS, D. Estudo comparativo entre elementos meteorológicos obtidos em estações meteorológicas convencional e automática em Botucatu, SP, Brasil. Revista Brasileira de Agrometeorologia, v.12, p.103-111, 2004.
DIAS, J.B. Construção e validação de uma bancada para calibração de sensores para determinação da umidade relativa do ar. 2001. 137p. Dissertação (Mestrado em Engenharia Mecânica) - Universidade Federal do Rio Grande do Sul, Porto Alegre.

DORCAS, M.E.; PETERSON, C.R. Automated data acquisition. In : G. Graeter and J. Gibbons (eds.), Monitoring Methods for Amphibians and Reptiles. Partners in Amphibian and Reptile Conservation - special publication. 2013.

FRITSCHEN, L.J.; GAY, L.W. Environmental instrumentation. New York: Springer-Verlag, 1979. 212p.

INCROPERA, F.; DeWITT, D. Fundamentals of heat and mass transfer. 5a ed., Edit. JohnWiley\&Sons. New York. 2003.

ISO - Internacional Organization for Standardization. Norma n.17714. Meteorology - air temperature measurements: test methods for comparing the performance of thermometer shields/screens and defining important characteristics, Geneva, 2007.

MARIN, F.R.; ANGELOCCI, L.R.; COELHO FILHO, M.A.; VILLA NOVA, N.A. Construção e avaliação de psicrômetro aspirado de termopar. Scientia Agricola, v.58, p.839-844, 2001.

PEARCY, R.W.; EHLERINGER, J.; MOONEY, H.A.; RUNDEL, P.W. Plant physiological ecology: field methods and instrumentation. Chapman and Hall, New York. 1989.

REYES CAC, F.E. Análise metrológica do comportamento transiente de um psicrômetro do tipo bulbo úmido e bulbo seco. 2005. 92p. Dissertação (Mestrado). Programa de PósGraduação em Metrologia para Qualidade e Inovação. Pontifícia Universidade Católica do Rio de Janeiro, PUCRio, 2005.

SILVA, C.R.; RAABE, J.; SILVA DIAS, N.; SILVA, T.J.A.; SANTOS, R.A. Desempenho de diferentes bitolas de termopar em um psicrômetro aspirado de baixo custo. Revista Caatinga, v.24, p.99-103, 2011.

WHITE, G.M.; ROSS, I.R. Humidity. In: HENRY, Z.A.; ZOERB, G.A.; BIRTH, G.S. Instrumentation and measurement forenvironmental sciences. Saint Joseph: ASAE, 1991. p.8.01-8.13

WMO -World Meteorological Organization. Guide to meteorological instruments and methods of observation, seventh edition, 2008. WMO n.8. Disponível em $<\underline{\text { http: }} / /$ www. wmo.int/pages/prog/www/IMOP/publications/CIMO_Guide/ CIMO\%20Guide\%207th\%20Edition,\%202008/CIMO_ Guide-7th_Edition-2008.pdf $>$. Acesso em 01 out. 2011.

WILLMOTT, C. J.; ACKLESON, S. G.; DAVIS, R. E.; FEDDEMA, J. J.; KLINK, K. M.; LEGATES, D. R.; O'DONNELL, J.; ROWE, C. M. Statistics for the evaluation and comparison of models. Journal of Geophysical Research, v. 90, p. 8995-9005, 1985. 